The success rate of those cases with less than three lashes per lid was $75.6 \%$ while the overall success rate was $62 \cdot 6 \%$. This is in accord with other authors' finding. ${ }^{2-4}$ Sharif ${ }^{3}$ explained this by the number of laser burns per lash. Our finding shows the association between lower recurrence rate and higher number of burns per lash: with no recurrence there were 16.7 burns per lash and with recurrence there were $14 \cdot 7$ burns per lash. However, this association cannot be proved statistically. Another possible explanation is that in those lids with high number of aberrant hairs the disease leading to trichiasis is still active and the hairs regrow after treatment.

YAT-MING YEUNG Tuen Mun Hospital,
Hong Kong

1 Gossman MD, Yung R, Berlin AJ, Brightwell JR. Prospective evaluation of the argon laser in the treatment of trichiasis. Ophthalmic Surg 1992; 23: 183-7.

2 Bartley GB, Lowry JC. Argon laser treatment of trichiasis. Am f Ophthalmol 1992; 113: 71-4.

3 Sharif KW, Arafat AF, Wykes WC. The treat ment of recurrent trichiasis with argon laser photocoagulation. Eye 1991; 5: 591-5.

4 Campbell DC. Thermoablation treatment for trichiasis using the argon laser. Aust $N Z \mathcal{F}$ Ophthalmol 1990; 18: 427-30.

\section{Electroretinogram as indicator of prognosis of central retinal vein occlusion}

EDITOR,-Matsui and colleagues ${ }^{1}$ report on ERG b/a wave ratio changes in central vein obstruction and conclude that retinal ischaemia in ischaemic central retinal vein occlusion (CRVO) can be reversible. This result is surprising, as the natural history of ischaemic CRVO has been clearly documented $^{2}$ correlating the degree of retinal ischaemia with the development of neovascularisation - a process that may only be reversed by panretinal photocoagulation (PRP). Their findings are based on investigations including fluorescein angiography (FA) and electroretinogram (ERG), both of which appear to require further analysis.

Firstly, with respect to the FAs in the good prognostic groups which supposedly illustrate resolution of retinal ischaemia. In case 3, the initial FA at 1 month was masked by retinal haemorrhages and the degree of ischaemia cannot be determined owing to the absence of peripheral photographs. Therefore, the FA at 5.5 months, said to show significant improvement, merely shows the predictable changes expected in a well perfused CRVO. Despite inaccuracies in statements regarding the timing of treatment and photography in case 1 (Fig 4), we feel there is no evidence of improvement of ischaemia in (according to the caption) the recovery FA at 5.5 months; this clearly demonstrates marked capillary drop out and macular ischaemia. It also demonstrates PRP which was supposedly not performed until 11 months.

Secondly, two different ERG techniques were used with only one set of normal values and no affirmation that each individual patient was always examined using identical techniques.

Thirdly, the ERGs in Figure 2 show a speculative b-wave identification on an ERG with no replication, and result in a markedly longer $a-b$ interval than when initially seen. If the b-wave were taken at the first visible peak, a very different amplitude value would be obtained. What were the criteria for b-wave identification?

Finally, following PRP, if case 1 stabilised, how do the authors explain such marked trial to trial variations in a- and b-wave amplitude (Table 1)? What is the expected intersession variability for their laboratory (normally about $10 \%$ )?

We therefore suggest that Matsui et al reconsider their results, or repeat the study with greater scientific stringency, and reassess the validity of their original conclusion.

$$
\begin{array}{r}
\text { M F CORDEIRO } \\
\text { M STANFORD } \\
\text { J S SHILIING } \\
\text { Greenwich District Hospital, } \\
\text { London SE10 } \\
\text { G E HOLDER } \\
\text { The Brook Hospital, } \\
\text { London SE18 }
\end{array}
$$

1 Matsui Y, Katsumi O, Sakaue H, Hirose T. Electroretinogram b/a wave ratio improvement in central retinal vein obstruction. $\mathrm{Br} \mathcal{F}$ Ophthalmol 1994; 78: 191-8.

2 Margargal LE, Donosa LA, Sanborn GE. Retinal ischaemia and risk of neovascularisation following central retinal vein obstruction ing central retinal vein
Ophthalmology 1982; 89: $1241-5$.

\section{Reply}

EDITOR,-We appreciate the comments of Cordeiro and others.

Firstly, they questioned our interpretation of the ischaemic CRVO in case 3, whose FA could not be interpreted because of blood covering the retina. Our interpretation of the ischaemic retina came mainly from the ERG findings of low b/a amplitude ratio with normal a-wave. The blood in front of the retina will not decrease the b/a ratio. It will increase the ratio instead. Non-ischaemic CRVO would not show decreased ERG b/a ratio. However, the level of retinal ischaemia in case 3 would be moderate because the decrease of the b/a ratio is not as marked as that usually seen in complete occlusion of the central retinal artery.

The fundus photograph in case 3 shows extremely dilated retinal veins, extensive retinal haemorrhage, and some capillary dropout in the area not covered by the blood in the FA. Cordeiro et al mentioned that the clearing of the haemorrhage and normalisation of the veins, as documented in case 3 , was a predictable outcome. I disagree. Predicting the final outcome of CRVO from the initial fundus appearance alone is difficult because one cannot judge the degree of retinal ischaemia if the retina is covered by blood. The ERG plays an important role in such cases.

There was an error in the caption for Figure 4. The FA in the middle of Figure 4 was taken after 11 months just before the PRP instead of 1 month. And the FA in the bottom was taken at 62 months instead of at $5 \cdot 5$ months. The captions of Figures 4 and 2 were mixed for which I apologise. Cordeiro et al feel that there is no evidence of improvement of the ischaemia after PRP (bottom Fig 4). The FA taken sometime after PRP was magnified in the posterior pole and shown in the bottom of Figure 4 . It is clear that the central non-perfused area is decreased after PRP compared with that before the PRP. The spot in the fovea in the bottom of Figure 4 is a pigmented scar spontaneously developed after the macular oedema subsided.

Secondly, Cordeiro et al asked why two different ERG techniques were used with only one set of normal values. This report is a retrospective study from data collected over many years. At one point we had to change the ERG system in our laboratory because of new recommended standardisation, and for other reasons. However, the majority (6/8 cases) had ERG recorded with the old system with description of the normal age matched values. Only two cases were recorded with the new system. ERG data used in those two cases were those recorded with bright single flash, strong enough to record oscillatory potential. The intensity was comparable with that of the old system. Only ERG b/a ratio was used in those two cases.

Thirdly, Cordeiro et al mentioned that the b-wave identification in Figure 2 was speculative. This criticism would be well taken if we had to measure the b-wave from this recording only. We routinely record ERG on EEG recording paper simultaneously with the cathode oscilloscope. In the reading on the EEG paper, whose paper speed is much slower, identification of the b-wave peak is easy. We recorded the amplitude from both these findings.

Finally, Cordeiro et al questioned if case 1 was stabilised, why was there a marked a- and b-wave amplitude variation after PRP. It would be wishful thinking that the retinal function would be stabilised after PRP. Obviously it was not. There was a change in the fundus appearance after PRP besides the scar formation on the laser spots, such as haemorrhage or vascular anastomosis during a long observation period after PRP, as described in the test. Vision fluctuated too. Therefore, it is not surprising to see some fluctuation of the ERG findings. In spite of this fluctuation, there was a trend in which the a-wave declined and the b-wave increased. As a result, the b/a amplitude ratio improved. The suggestion was made to repeat the study with greater scientific stringency. Such a study requires a long time if done by one institute. A nationwide study in CRVO has been recently completed in the United States in which multiple medical centres participated. This study included ERG and FA. The results of the study may disclose a similar case. Namely, ischaemia in CRVO may not be permanent and may be reversible in some. T HIROSE
O KATSUMI Shepens Eye Research Institute, 100 Charles River Plaza,
Boston, MA 02114, USA

Effect of trabeculectomy on pulsatile ocular blood flow

EDITOR,-We read with interest the paper by James. ${ }^{1}$

An increase of pulsatile ocular blood flow (POBF) was found in the standing position following trabeculectomy. This was attributed to an increase in perfusion pressure which is expected with reduction of intraocular pressure (IOP) assuming autoregulation was absent.

In the lying position, however, POBF was unchanged following trabeculectomy despite similar magnitudes of IOP lowering. It was suggested that in this group, because of the extremely high IOP in the lying position preoperatively, the POBF was somehow maintained at an elevated level by autoregulatory mechanisms which masked any improvements in POBF after surgery. This was also felt to be responsible for the regaining of the usual postural changes following trabeculectomy. 